\title{
The Use of Art in the Teaching Practice for Developing Communication Skills in Adults
}

\author{
Niki Phillips \\ Tutor, Hellenic Open University, Patra-Greece \\ Head of Human Resource Development \\ Bank of Cyprus, Greece \\ Tel: 30-694-568-1885Ｅ-mail: niki.phillips@gmail.com
}

Iosif Fragoulis

As. Professor, School of Pedagogical and Technological Education

Tutor, Hellenic Open University, Patra, Greece

Tel: 30-261-091-0066 E-mail: sfaka@otenet.gr

Received: September 13, 2011 Accepted: October 19, $2011 \quad$ Published: April 1, 2012

doi:10.5539/ies.v5n2p132

URL: http://dx.doi.org/10.5539/ies.v5n2p132

\begin{abstract}
The use of Art for educational reasons has been recently developing in Greece both in formal education and in Adult Education. Relevant theoretical texts and studies, (Dewey, 1934. Gardner, 1990. Perkins, 1994) pin point that training through the Arts can contribute to an integrated learning, since through systematic observation of works of art, the trainees' critical thinking, creativity and fantasy can be generated.

The first part of the current paper, examines the reasons that necessitate the use of art in the training practice.

The models of approaching and understanding art for educational reasons, as presented and analyzed by Feldman, Brondy, Anderson and Perkins, are presented in the second part.

The method "Transformative Learning through an aesthetical experiences", has been grounded and developed by A. Kokkos, and is presented in the third part. The different stages of this method are also analyzed.

In the final part, an example of Kokkos' method is being analyzed (stage by stage), regarding the training for an organization's staff development of their communication skills.

Conclusions regarding the use of art in the training praxis, may be found at the final part of this paper.

Keywords: Art in training, Formal training, Teachers' training, Adult trainers, Transformative learning, Communication skills, Critical reflection, Cognitive development

\section{Introduction}

In recent years an educational format based on the exploitation of art for training purposes, has been developing both in formal and non formal education fields.

Many theorists such as Gardner, Dewey, Perkins, Kokkos, have been expressing the view that education and training through art pieces can positively contribute to the learning process and help trainees develop various competences such as critical thinking, creativity, emotional expression (Kokkos, 2011).

Although one can encounter in formal education many examples of art exploitation during the teaching praxis, in the adult education field very few references can be found regarding the use of art in training, such as the ones mentioned in Kokkos' book "Training through Art".

Systematic efforts of using art in the training of teachers of formal education are currently being made in Greece under the implementation of the project "Major Training Program". Similar initiatives have been taken by the Scientific Association of Adult Education during the training of adult trainers.
\end{abstract}

\section{The Need of Using Art in the Teaching Praxis}

Many Adult Education theorists (Perkins, 1994. Kokkos, 2011. Mega, 2011), argue that the development of the 
reflective process through the observation of art can be initiated through specially tailored actions (Kokkos, 2011:21). Such actions may have the following aims:

A. The creation of conditions for effective learning through the trainers' and trainees' active participation during the observation of works of art

B. The trainers' and trainees' realization that culture and its creation should not be excluded from the daily training practice, but must be an important part of it

C. The development of the trainers' and trainees' reflective attitude

D. The development of the participants' in the training process (both trainers and trainees) holistic systemic thinking (Kokkos, 2011:22).

According to Perkins (1994) contact with works of art promotes critical thinking (Kokkos, 2011:39). This argument is presented in his monograph titled "The intelligent eye: Learning to Think by Looking Art" (Perkins, 1994). He mentions that art works as "... an anchor for the senses" since art assists in focusing to a particular situation or event during the learning process.

Kokkos (2011) supports that arts contain dynamically informing data which reinforce the sensory perception broaden culture and built a cultural awareness (Kokkos, 2011:39).

Esnerr (2002) states that observation of art is associated with cognitive development, since it contributes to observation growth, activation of imagination and flexibility of thinking (Esner, 2002:35-42).

Elfand (2002) considers art analysis as a mean for the activation of the trainees' reflective temper. Careful observation of works of art can lead thinking towards various extensions: social, political, historical, ideological etc. Such multi linked thinking can develop the observer's critical disposition (Kokkos, 2011: 41).

Additionally, through art observation one may recall powerful personal experiences. Such memory recall can activate reflection which can lead to reprocessing those experiences. Reflection of experiences leads to metacognition (Kokkos, 2011: 42).

\section{Models Approaching Art in the Training Process}

Models approaching art provide a theoretical and methodological context according to which trainees gradually understand a work of art. They also can enter areas related to a deeper interpretation while their critical and reflective attitude is being activated (Kokkos, 2011: 44).

In relevant bibliography four such models are being described: Feldman's, Broudy's, Anderson's and Perkins'. According to Feldman (1967) understanding of art can be achieved through four interrelated phases: description, analysis, interpretation and evaluation.

Broudy's model (1972) - which is similar to the Feldman model - focuses in the activation of the sensory observations of an art work (e.g. search of patterns, volume, their connections etc) as well as in identifying the expressiveness exuded by the piece of art.

Anderson's model (1993) includes the following reflective processes: initial reaction, description, interpretation, evaluation. In many ways it resembles the Feldman's model, however it differs from it in the first phase of the initial reaction. In this phase the trainee proceeds to an instinctual reaction towards the work of art being observed.

Perkins' model is considered to be the most complete in relation the other three models mentioned here, since it may - through minor modifications - be applied to the approach of various pieces of art (Mega, 2002. Kokkos \& Mega, 2007. Kousoulas \& Mega, 2005).

According Perkins' context of understanding art, the viewers adopt a documented glance towards the observed art. They are not only limited to evaluative judgements such as "like", "do not like", but they orderly develop an argument which enhances their perspective for the particular work of art (Kokkos \& Mega, 2007: 17).

Perkins supports that such process assists trainees to enhance their sensory experiences, while generating a critical/reflective attitude towards the events taking place within and outside the training context (Perkins, 1994: 87-89).

Perkins' model consists of four phases which are interrelated and can be summarized as follows:

\section{First phase: Time for observation}

The viewers carefully observe a piece of art without attempting to understand or evaluate it. They aesthetically respond to the subject matter. 


\section{Second phase: Open and adventurous observation}

During this phase the viewers observe to work of art with an open mind and a creative attitude. They carefully observe while having in mind "what is that the artist would like us to observe".

\section{Third phase: Analytical and deeper observation}

The trainees utilize the data collected in the previous stages in order to deeply understand art. They attempt to answer the questions which arose in the two previous phases. They also try to interpret, substantiate their opinions and draw conclusions.

\section{Fourth phase: Review of process}

During the fourth phase trainees holistically approach the work of art, by exploiting the observational experience of the previous stages. They reflect and proceed to the writing of a text regarding what they have experienced during observation, focusing on their thinking transformations they detect, their assumptions and hypotheses in relation to the subject matter.

\section{The Method "Transformative Learning through Aesthetical Experiences"}

This method is introduced by A. Kokkos (2010) and functions in the Transformative Learning theoretical context. The development of this method is based on the existence of a "disorienting dilemma" which leads the trainees to the re-evaluation of their assumptions in relation to a subject matter (Kokkos, 2010).

The observation of art from the perspective of a particular content enhances the trainees' critical reflection and the critical evaluation of their assumptions in regards to the particular subject. The main aim of this process is to transform the trainees' frame of reference, hence their complete thinking structure.

The method is implemented through the following six interrelated stages:

\section{First stage: Exploring the need for critical reflection}

During this stage the trainer explores the trainees' need for critical reflection upon their intellectual habits regarding a subject matter. Such need can be detected either at the commencement and/or during the implementation of a training program. The need is recognized by the trainer who asks the learners whether they desire to systemically approach the particular matter during their training sessions.

\section{Second stage: Participants express their views}

The participants' views regarding a particular matter are systematically recorded. Their views are firstly discussed within smaller teams followed by a plenary discussion session.

\section{Third stage: Definition of views to be discussed}

During this stage both the trainer and the trainees co decide for the views to be discussed and to be critically reflected upon, as well as the order of matters to be analyzed.

\section{Fourth stage: Selection of works of art for the views approach}

The trainer cooperates with the trainees for the selection of those works of arts which can serve as a trigger to the approach of the views selected to be analyzed.

\section{Fifth stage: Critical reflection through the aesthetic experience}

At this stage the trainer coordinates the trainees' critical reflection process, by exploiting the Perkins' model of responding to and understanding art.

\section{Sixth stage: Re evaluation of assumptions}

During this final stage the trainees' assumptions - in relation to the work of art - are being re evaluated. The final assumptions are compared to those initially expressed by the trainees.

\section{Description (Note 1) of A Practical Example for the Exploitation of the Method "Transformative Learning Through Aesthetical Experiences".}

The following example could be applied in the training program: "Effective Communication Skills" under the topic "The importance of body language". Total duration estimated for its completion: 2 hours (120 minutes).

\section{First Stage: Exploring the need for critical reflection $20 \mathrm{~min}$}

- Participants are asked:

- How important do you consider Body Language for your everyday communication? 
- Disorienting dilemma: The Albert Mehrabian research is presented. According to this research the meaning of the message we wish to convey, is subject - up to $7 \%$ - to the words we use, while the rest of the $93 \%$ depends on the way we express them (voice, gestures etc).

- The trainer asks the participants to think - during the following stages of this activity - about whether the Mehrabian's research findings are too exaggerated.

- The trainer asks the trainees to individually record on a piece of paper, a thought (maximum one line long) and a sentiment (using one word) regarding the procedure that will follow (the 6 stages) in relation to the importance of body language. He/she tells them to keep aside the piece of paper for later use.

\section{Second Stage: Participants express their views $15 \mathrm{~min}$}

- The trainer asks the participants to discuss in groups of 4-5 members the following open ended question:

- What does "I understand Body Language" mean?

- The group representatives announce their answers (eg. gaze, facial expressions, poise, gestures, distances between people etc), thus the trainer discovers the way they interpret the meaning of the term "Body Language".

- The trainer needs to discover whether participants know the following basic advantages and benefits for those who are able to interpret and understand body language:

- Reinforcement of interpersonal relationships

- Control over own body language and the way perceived by others, during communicational transactions.

- Development of persuasion skills

- Ability to interpret and understand other people's body language of

- Recognition and interpretation of the hidden messages of people with whom they communicate.

- Depending on the teams' answers, the trainer decides upon the points they should focus during the rest of the training session.

\section{Third stage: Definition of views to be discussed $10 \mathrm{~min}$}

- The trainer asks from each team to write down one key question that arose during their second stage discussion "What does I understand Body Language mean?"

- On a flipchart titled "What does I understand Body Language mean?" the trainer notes down the key questions announced by the teams' representatives.

- The trainer announces that the main aim of the activities and discussion which will follow is to answer to the written questions noted on the flipchart and hanging in the training room.

\section{Fourth Stage: Selection of the works of art}

- The trainer has shortlisted three works of art (see appendix) which assist in the study and analysis of body language's importance. These are:

- $\quad$ Amedeo Modigliani - Portrait of a Young Woman 1

- Charlie Chaplin - excerpt (detail from 4:15-6:18) from the film "City Lights"

- Photograph awarded with the "Pulitzer Breaking News Photography 2007 award"

- Initially the Modigliani painting will be exploited and in a second phase the other two art works will be analyzed. The Modigliani figures - like the Portrait of a Young Woman 1 used in this activity - are motionless, with no distinct eyes and faces which assimilate masks. Thus, body language elements are absent.

\section{Fifth Stage: Critical reflection through the aesthetic experience 60 min (total time of $5^{\text {th }}$ stage).}

In this stage, the phases of the Perkins' model are followed, as described below:

First phase: Time for observation $10 \mathrm{~min}$

The Modigliani painting is displayed and participants are asked to individually complete the phrases: "I see.... $I$ think that... I wonder about..."

Then they freely express in a plenary session the thoughts they wrote under the phrase "I wonder about ..." while the trainer, summarizes (and categorizes if possible) the questions which arose. 


\section{Second phase: Open and adventurous observation $20 \mathrm{~min}$}

The trainees work in groups (four or five). Each group elaborates on one of the following issues:

- Suppose you hear the voice of the woman. How do you imagine it to be and why?

- How would the painting look if you could draw the woman's facial expressions?

- What would change if you could see the woman's look and gestures?

- Suppose you see the woman's body move. What does it look like and why? (more similar questions can be asked).

\section{Third phase: Analytical and deeper observation $10 \mathrm{~min}$}

Each group's representative announces to the plenary the group's answers. The trainer assists the participants through discussion to deeply interpret and understand the painting. (Note 2)

\section{Fourth phase: Review of process 20 min}

The trainees reflect on the painting with the trainer aiming to emerge the fact that the analysis of the body language can alter the interpretation of the painting and give a different dimension to it.

The analysis of the other two works of art is followed:

- Charlie Chaplin - excerpt (4:15-6:18) from the film "City Lights":

The participants watch the particular part of the film and discuss in plenary the meanings of the actors' body language that can be transmitted.

- Photograph awarded with the "Pulitzer Breaking News Photography 2007 award":

Participants observe the photograph and discuss in plenary what they would not be able to detect if they only red the particular news without being able to see the photograph.

\section{Sixth Stage: Re evaluation of assumptions $10 \mathrm{~min}$}

In this stage the trainer asks the question: "Compared to the beginning of our session, what new ideas and views do you now have about body language?" The trainer encourages participants to compare in groups their present views regarding body language in comparison with the views expressed on the Third Stage (the relevant questions are still posted around the classroom).

\section{Next Stages: 5 min}

- The trainees individually return to the information (a thought and a feeling) they had written down on a piece of paper during the First Stage. They now write a new thought and a new feeling regarding body language.

- The trainer coordinates a relevant discussion and summarizes their views, compounding all of the body language's basic elements (such as gestures, body movements, eye look, facial expressions, etc).

From the process described in the above mentioned stages the trainer seeks and aims at the participants' transformation of assumptions regarding Body Language and its importance for one's communication abilities.

\section{Conclusions}

From the above presentation of the theoretical approaches in regards to the use of art in the training praxis the following conclusions can be drawn:

The aesthetic experience gained by approaching and understanding works of art, contributes to the generation of the adult trainees' multiple intelligences, since the art symbols carry their own particular characteristics (Gardner, 1990:10).

Contact with works of art promotes reflection and assists in the development of critical thinking (Perkins, 1994), which is considered as the basic element of adult learning.

Contact with works of art cultivates the trainees cultural personalities, delineates the cultural and historical context of each era while it recognizes the coexistence of different cultures (Fowler, 1996:55).

Works of art provide us with rich material which enhances our sensory perception, broadens culture and develops cultural awareness. Careful and detailed observation of art gives information regarding social values, historical and cultural data, philosophical views and aesthetical habits of an era during which the particular art work was created (Kokkos, 2011:39).

Observation of art work leads human thinking into various social, political, ideological, and aesthetical extensions. 
The interrelation of such extensions contributes to the development of the adult trainees' critical attitude (Kokkos, 2011: 41).

For effective use of art in the training praxis, the systematic training of adult trainers in relation to such methodological approach should be implemented. Until today, this task has been undertaken in Greece by the Scientific Association of Adult Training, which designs and delivers relevant training programs in many of the largest Greek cities. It also delivers lectures in Post Graduate Courses of the Hellenic Open University, the University of Patras and the University of Macedonia.

It is also proposed the delivery of a training program targeted to adult trainers which will focus on the "educational exploitation of works of art", by the use of a blended method (distance training and in classroom training).

\section{Acknowledgements}

We express our appreciation to Ms Dora Aggelopoulou (Msc, in HR Management), trainer/facilitator at the Bank of Cyprus, Greece, for her permission to use extracts from a training program she personally delivers for the bank employees, and focuses on the interpretation and understanding of body language.

The aim of this activity is to help trainees understand the "static" character of the painting and its deeper interpretations, by the comparison of the "I wonder..." statements with the austerity and simplicity of the artwork.

\section{References}

Eisner, E. (2002). The Arts and the Creation of Mind. New Haven and London: Yale University Press.

Fowler, Ch. (1996). Strong Arts, Strong Schools. London: Oxford University Press.

Gardner, H. (1990). Art Education and Human Development. California, Los Angeles: The Getty Education Institute for the Arts.

Kokkos, A., \& Mega, G. (2007). "Research Project in Process: Critical Reflection and Art in Education". Adult Education, 12, 16-21. (In Greek)

Kokkos, A., \& Associates. (2011). Training Through Arts, Athens: Metaixmio (In Greek).

Kousoulas, F., \& Mega, G. (2005). Language and Art: Proposal for Creative Teaching. A Research on Primary School Student, Educational Survey, 40,120-133. (In Greek)

Mega, G. (2002). The Arts as an Effective Learning Resource: The example of teaching language. Unpublished Doctoral Dissertation, University of Crete, School of Education, Rethimno, Greece. (In Greek)

Mega, G. (2011). Arts in Schools as a Reflective Process. In A. Kokkos, \& Associates (Eds.). Training Through Arts, (pp.21-67). Athens: Metaixmio. (In Greek)

Perkins, D. (1994). The Intelligent Eye: Learning to Think by Looking at Art. California, Los Angeles: The Getty Education Institute for the Arts. 


\section{Appendix}

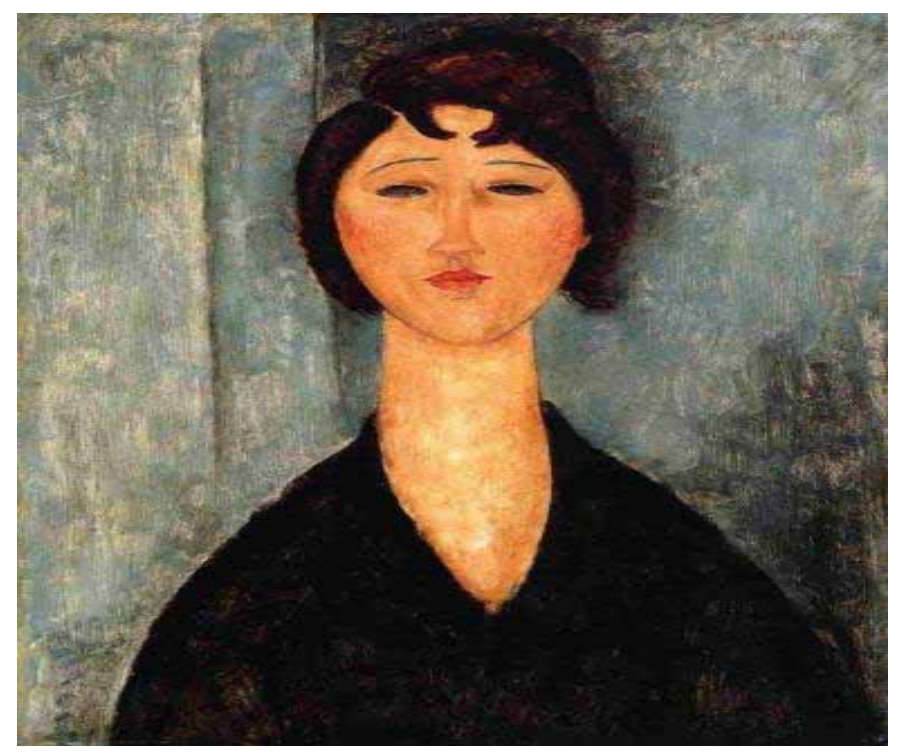

Amedeo Modigliani - Portrait of a Young Woman 1 (Oil Painting)

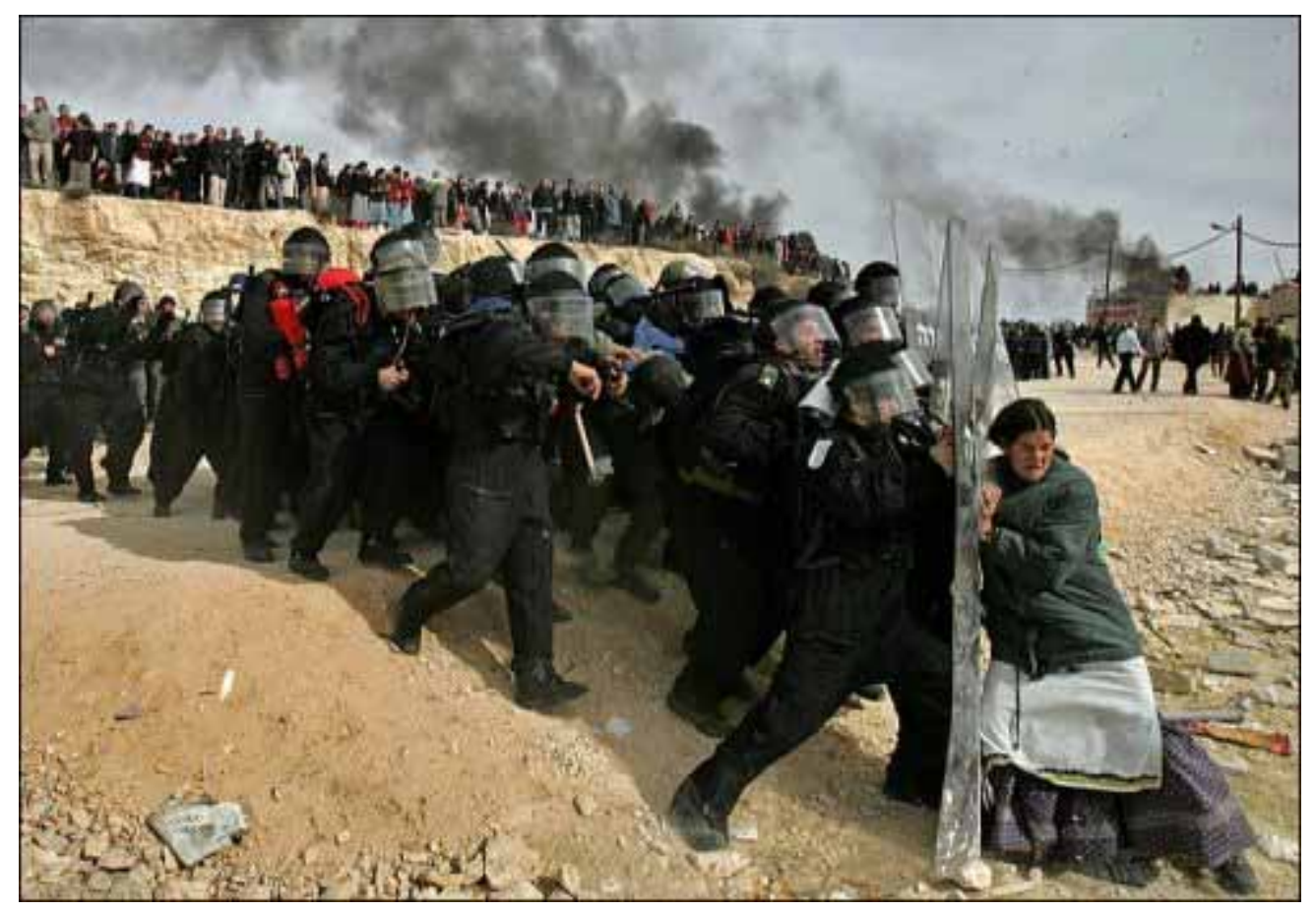

Pulitzer Breaking News Photography 2007 award

"Lone Jewish woman defying Israeli security forces as they remove illegal settlers in the West Bank" Photographer: Oded Balilty (Associated Press) 